\title{
Retrosigmoid Craniectomy for Resection of Epidermoid causing Trigeminal Neuralgia
}

\author{
Anubhav G. Amin ${ }^{1}$ John V. Wainwright ${ }^{1} \quad$ Chirag D. Gandhi ${ }^{1} \quad$ Christian A. Bowers $^{1}$ \\ ${ }^{1}$ Department of Neurosurgery, Westchester Medical Center Health \\ Network and New York Medical College, Valhalla New York, \\ United States \\ Address for correspondence Anubhav G. Amin, MD, Department of \\ Neurosurgery, Westchester Medical Center Health Network and \\ New York Medical College, 100 Woods Road, Macy Pavillion, Valhalla, \\ NY 10595-1696, United States (e-mail: anuamin@gmail.com).
}

J Neurol Surg B 2019;80(suppl S3):S320-S321.

\begin{abstract}
The differential diagnosis for trigeminal neuralgia like-symptoms includes cerebellopontine angle lesions causing regional mass effect upon the trigeminal nerve (-Fig. 1). Here we present an operative video manuscript of a patient experiencing trigeminal neuralgia, secondary to an epidermoid cyst, in which a retrosigmoid craniectomy was performed to resect the epidermoid and decompress the trigeminal nerve (-Fig. 2). This video highlights the operative nuances to achieving a successful surgery, including

\section{Keywords}

- epidermoid cyst

- trigeminal

- neuralgia

- technique appropriate patient positioning, dural exposure to the transverse-sigmoid sinus junction, arachnoid dissection, and decompression of cranial nerves. A gross total resection was achieved; the patient reported immediate relief of facial pain postoperatively and has been pain free at the ten month follow-up. The link to the video can be found at: https://youtu.be/Ja2eE0uGk4E.
\end{abstract}

Conflict of Interest None.

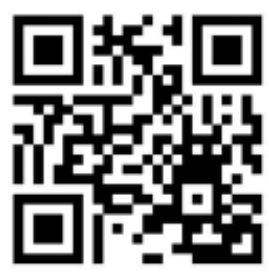

received

June 1, 2018

accepted

November 11, 2018

published online

February 18, 2019 www.thieme.com/skullbasevideos

www.thieme.com/jnlsbvideos

DOI https://doi.org/

10.1055/s-0038-1676998. ISSN 2193-6331. (c) 2019 Georg Thieme Verlag KG
Stuttgart · New York

License terms

(c) $\odot \ominus$ 

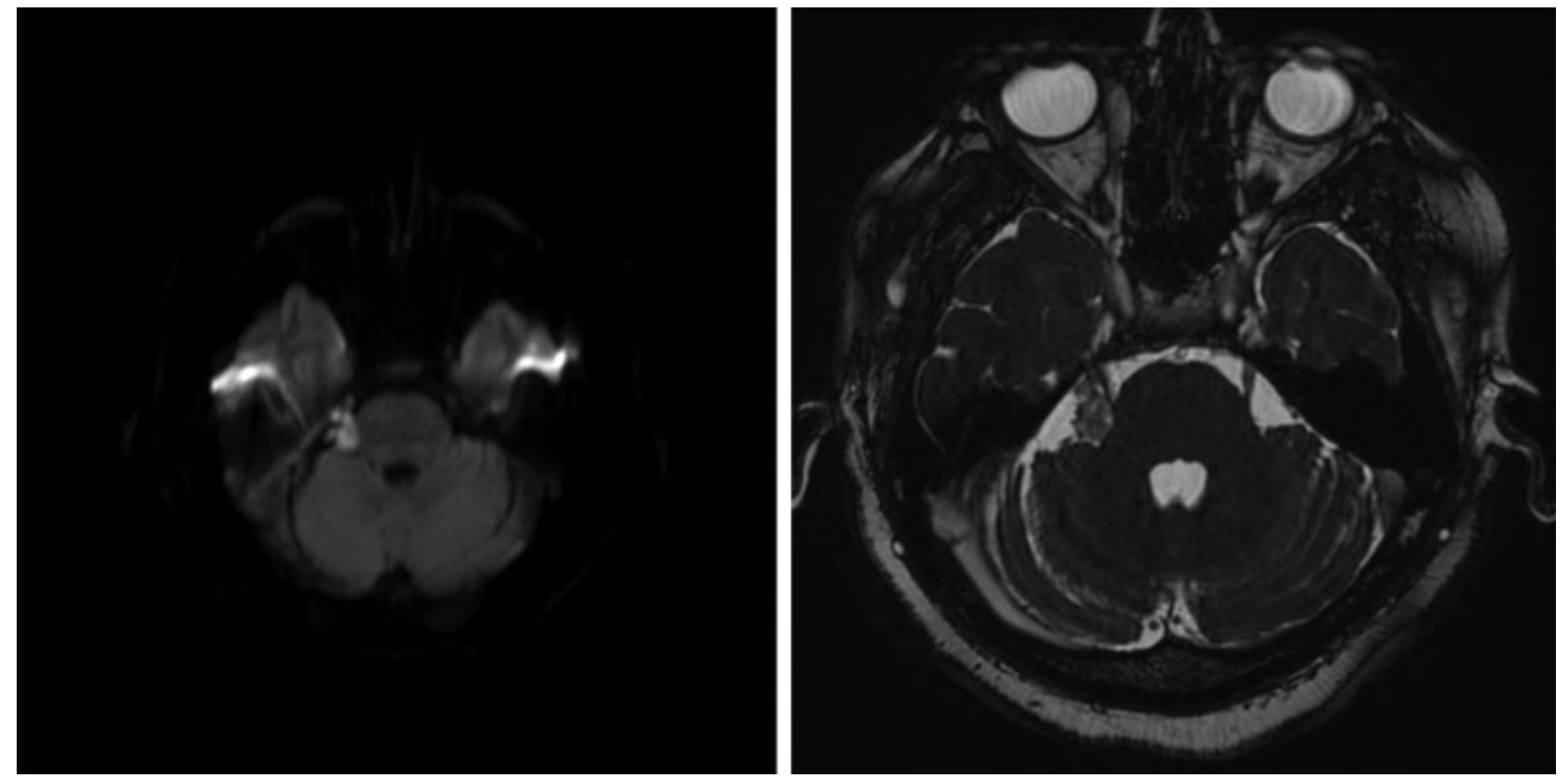

Fig. 1 Axial diffusion weighted and axial T2 B-FFE MRI demonstrating a diffusion restricting lesion in the right cerebellopontine angle compressing the root entry zone of the right trigeminal nerve and indenting the pons. B-FFE, balanced fast field echo; MRI, magnetic resonance imaging.

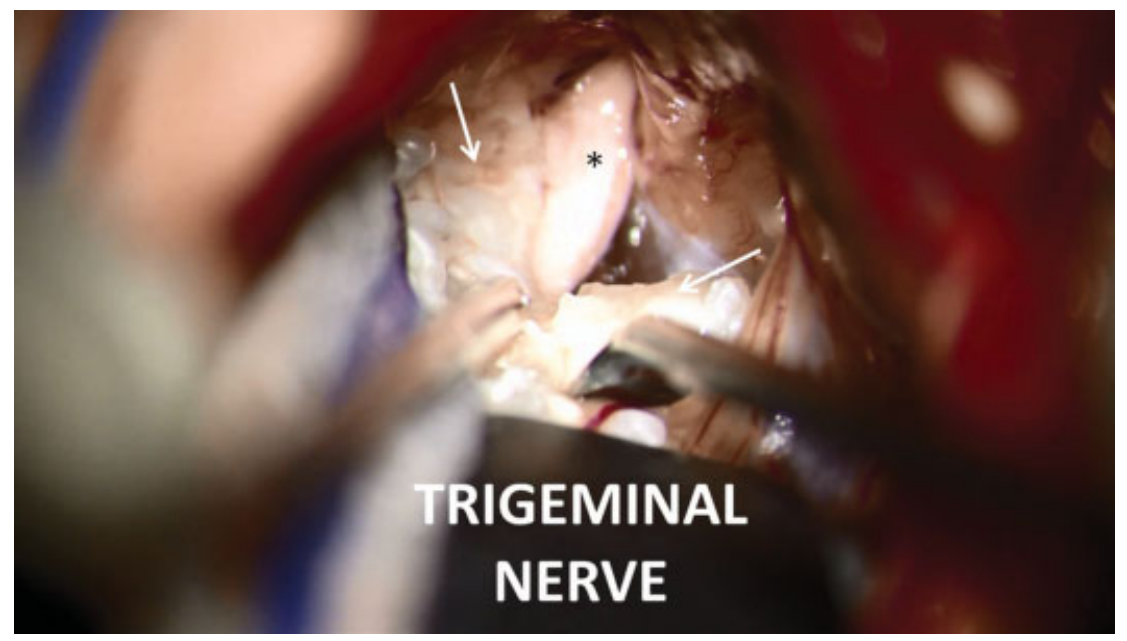

Fig. 2 A right retrosigmoid craniectomy demonstrated a pearly white, friable lesion (arrows) compressing and encasing the trigeminal nerve ${ }^{*}$ ) that was diagnosed as an epidermoid cyst on pathology. 